\title{
Research Paper: The Relationship of Work Ethics With Job Performance of the Administrative and Medical Staff of the University of Social Welfare and Rehabilitation Sciences and Its Affiliated Centers in 2017
}

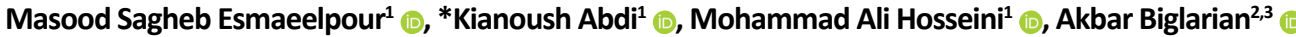 \\ 1. Department of Rehabilitation Management, University of Social Welfare and Rehabilitation Sciences, Tehran, Iran. \\ 2. Department of Biostatistics, University of Social Welfare and Rehabilitation Sciences, Tehran, Iran. \\ 3. Social Determinants of Health Research Center, University of Social Welfare and Rehabilitation Sciences, Tehran, Iran.
}

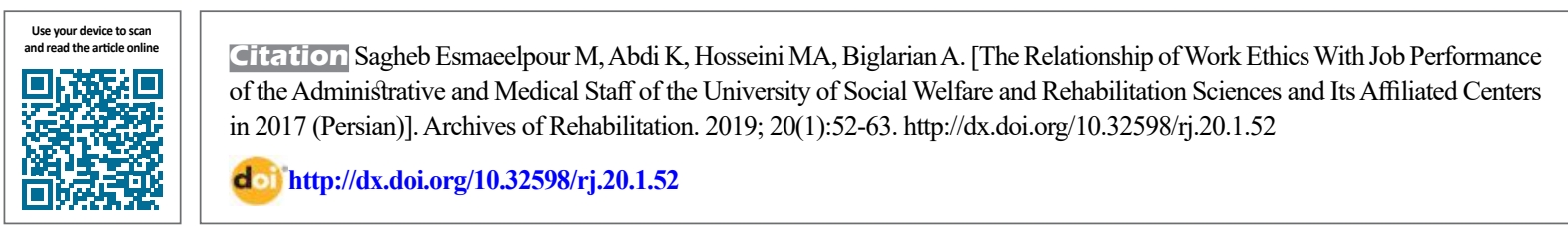

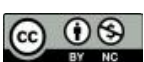

Received: 05 Dec 2018 Accepted: 10 Mar 2019 Available Online: 01 Apr 2019

Keywords:

Job performance, Work ethics, University of Social Welfare and Rehabilitation Sciences, Affiliated centers

\section{A B STRACT}

Objective The most effective and influential capital of any organization is its human resources. The promotion of financial and physical resources results from human resource development. Recognizing and identifying the factors affecting job performance is one of the important management tasks on the macro scale. Therefore, its related factors such as recruiting, training, educating, and maintaining employees, are planned and implemented in a better way by human resource management. If ethical values such as commitment to work, honesty, and integrity are observed, the organization's job performance and success will increase. The present study was conducted with the aim of explaining the relationship between work ethics and job performance of the administrative and medical staff of the University of Welfare and Rehabilitation Sciences and its affiliated centers in 2017

Materials \& Methods This was a descriptive-analytic research study. The study population was the entire 1506 administrative and medical staff of the University of Social Welfare and Rehabilitation Sciences and its affiliated centers (Razi Psychiatric Hospital, Rofeideh Rehabilitation Hospital, and Mafi Rehabilitation Center) in 2017. Out of their staff, a total of 250 employees were selected by the straight randomized methodas the study sample. The research instrument consisted of a valid and reliable questionnaire with 15 questions about Patterson's job performance, 50 questions about Gregory's criterion work ethics that has 4 dimensions (attachment and interest, seriousness and perseverance, healthy relationships, and collective spirit and participation), and a demographic information questionnaire. To analyze the data, in the descriptive statistics section, indicators such as statistical tables, distribution indices, and percentage, and in the inferential statistics section (with regard to the abnormal distribution of data) non-parametric tests of Spearman's correlation coefficient and Mann-Whitney U test were used.

Results The results showed a significant positive correlation between work ethics and its dimensions with the job performance of employees $\left(P<0.001, R^{2}=0.366\right)$. There is a correlation coefficient of 0.566 between attachment dimension and the interest of work ethics with job performance. The correlation coefficient of the persistence of ethics with job performance is 0.556 . The correlation coefficient of the relationship of healthy work ethics with job performance is 0.512 and the amount of the correlation coefficient of the collective spirit of work ethics with job performance was 0.570 . Therefore, there is a significant relationship between all aspects of work ethics and job performance $(P<0.001)$.

Conclusion Regarding the direct relationship between work ethics and job performance, the promotion of employees' morality is associated with an increase in employees' job performance. For this purpose, it is suggested that the attention to work ethic in various aspects of epistemic programs and organizational learning be increased, in order to increase the performance and active participation of employees in organizational matters and to perform their responsibilities. The principals of the university and its affiliate health centers must also pay attention to the various ethical dimensions of the administrative and medical staff in order to increase the level of their employees' job performance and try to raise the moral level of employees by holding workshops, forming problem-solving teams, and setting up a reward system for their employees.

\section{* Corresponding Author:}

Kianoush Abdi, PhD.

Address: Department of Rehabilitation Management, University of Social Welfare and Rehabilitation Sciences, Tehran, Iran.

Tel: +98 (21) 22180132

E-Mail: k55abdi@yahoo.com 


\title{
رابطه اخلاق كارى با عملكرد شغلى كاركنان ادارى درمانى دانشخًاه علوم بجزيستى و توانبخشى و

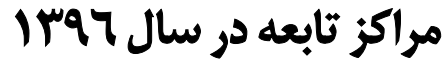

\author{
مسعود ثاقب اسمعيليوره' ، 'كيانوش عبدىه' ، محمد على حسينىه' ، على اكبر بيكلريانه ‘r.

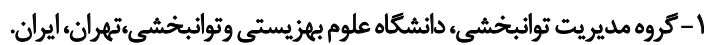

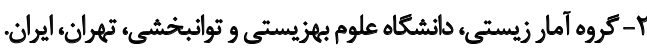

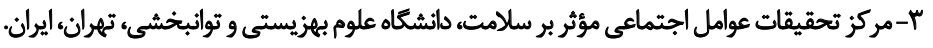

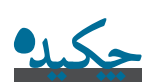

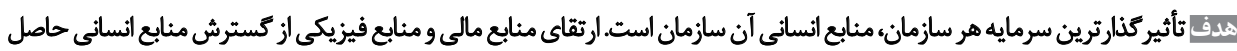

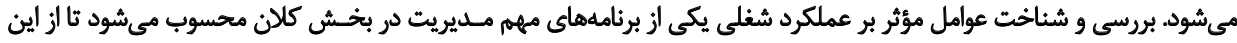

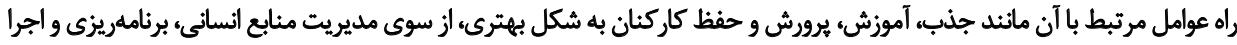

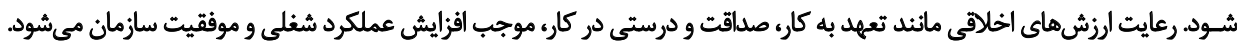

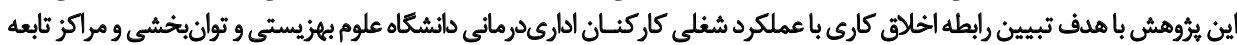

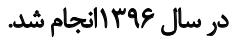

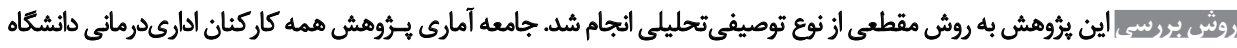

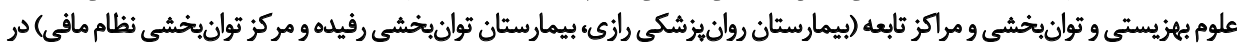

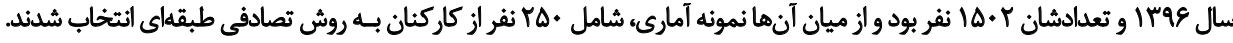

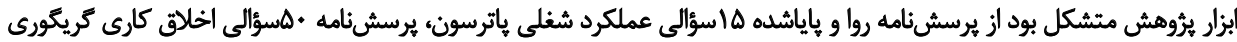

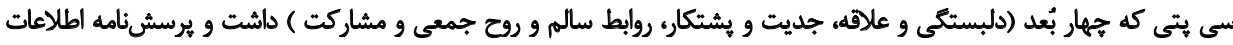

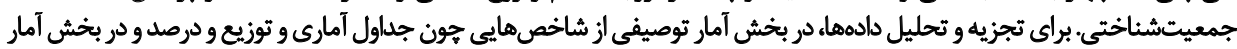

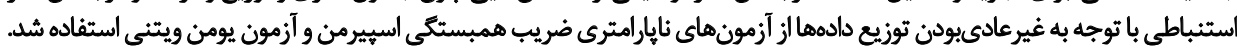

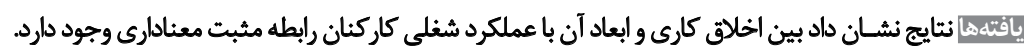

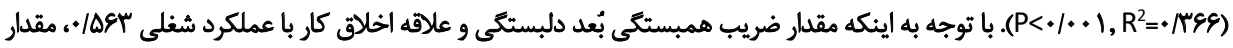

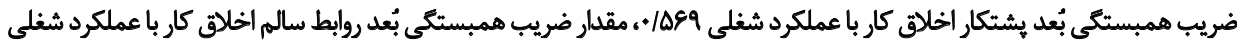

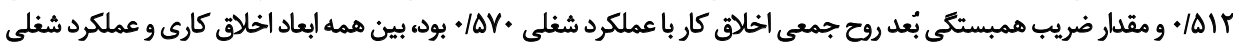

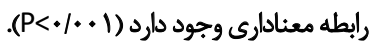

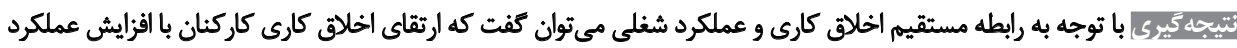

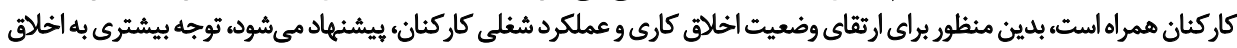

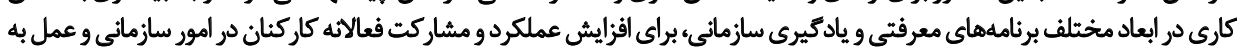

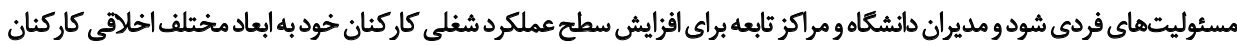

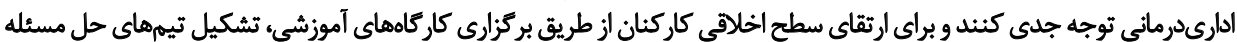
ونظام تشويق كاركنان خود بكوشند.
\end{abstract}

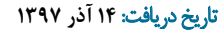

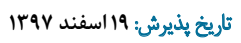

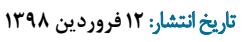

كليدوازوهها: عملكرد شغلى، اخلاق كاري، دانشّاه علوم

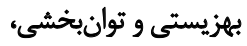
مراكز تابعه 
دغدغههايي كه در قالب يك مسئله مطرح مىشوده اين است

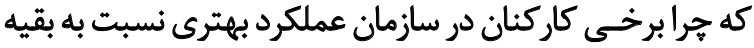

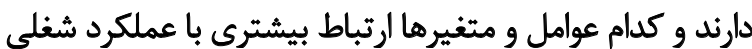

دارند [ارند

عوامل مختلفى ميىتواند روى عملكرد شغلى كاركنان و ورئي

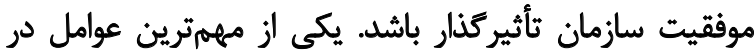

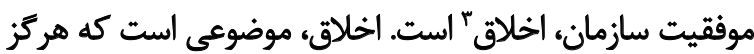

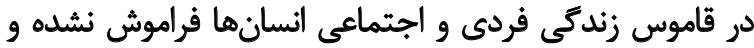

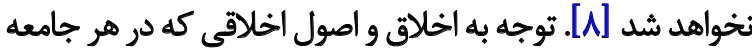

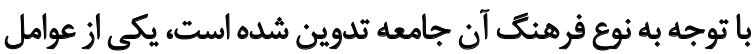

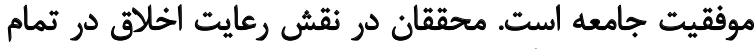

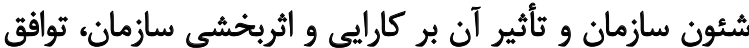

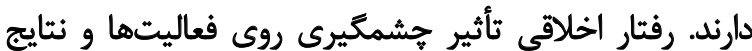

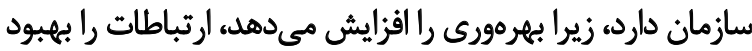

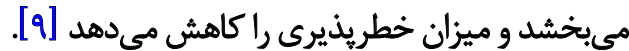
در دنياى مدرن امروز مفاهيم اخلاق كارى و مسئوليتهاى

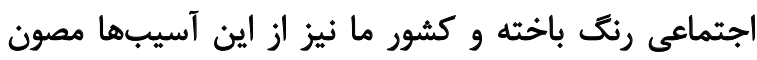

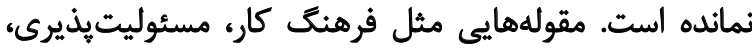

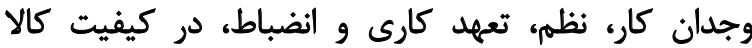

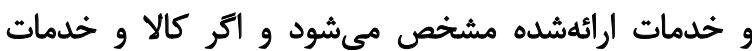

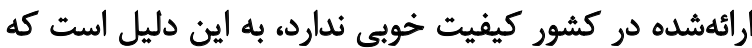

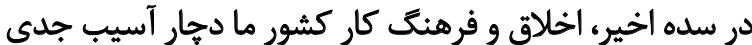

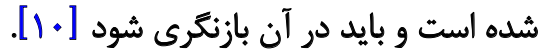

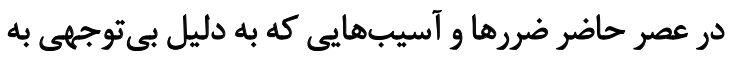

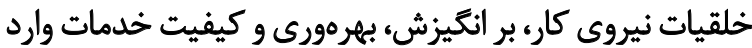

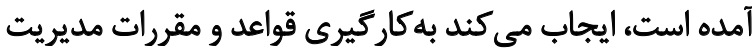

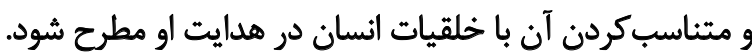

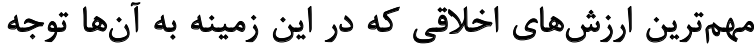

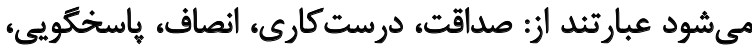

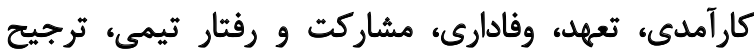

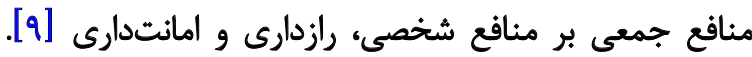

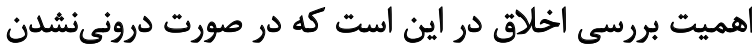

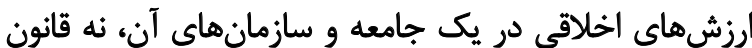

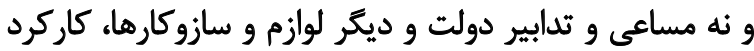

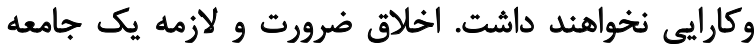

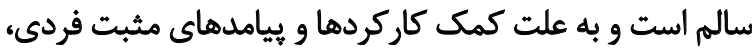

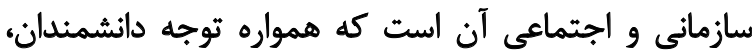

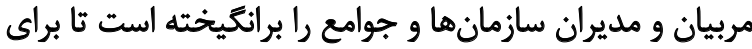
حفظ و ارتقاى سطح آن كوشش كنيند.

اخلاق كارى يكى از مسائل اساسى همه جوامع بشرى است.

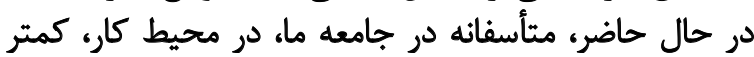

3. Ethic olad

در تمام ديدكاهها، مكاتب و الكوهاي توسعه اجتماعى، از باز

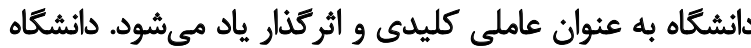

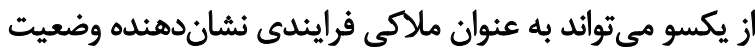

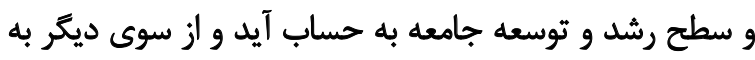

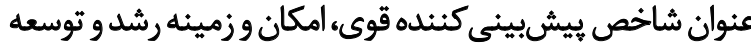

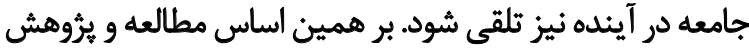

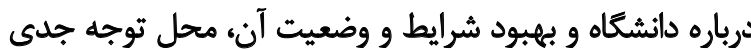
برنامهريزان و مسئولان اجتماعى بوده است ـ [1]

سازمانها براي استمرار و استقرار خود با جالشهاي نوينى

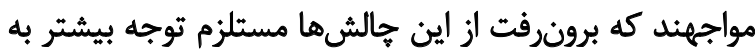

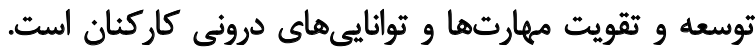

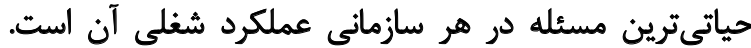

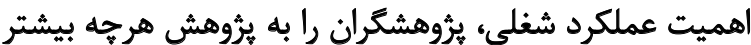

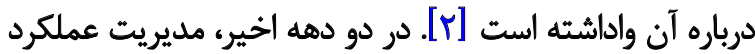

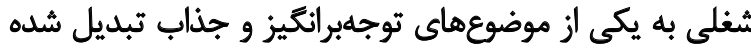

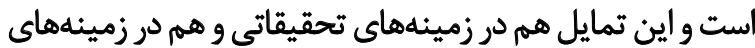

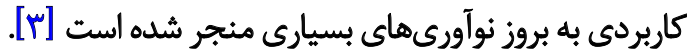

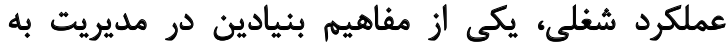

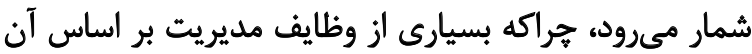

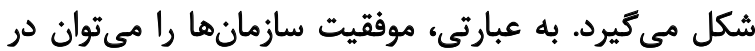

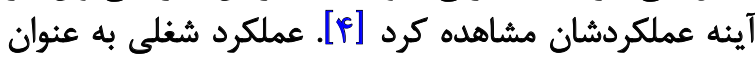

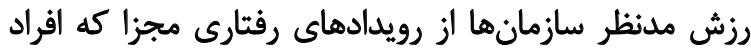

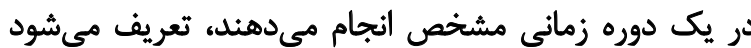

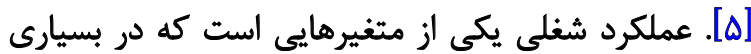

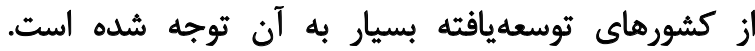

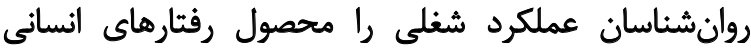

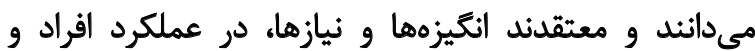
درنهايت رشد و توسعه اقتصادى تأثير دارند [عانداند. در حقيقت عملكرد شغلى با توجه بـهـ مبنـاى نظرى موجود در

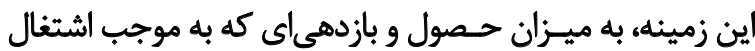

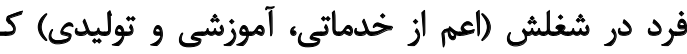

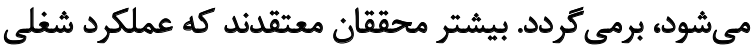

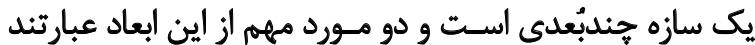

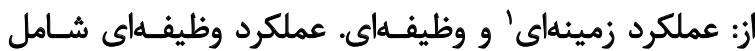

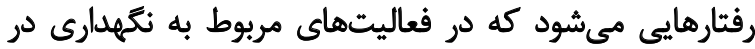

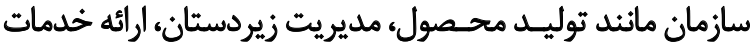

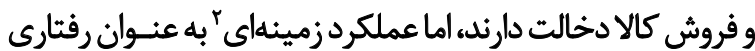

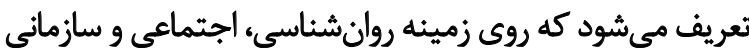

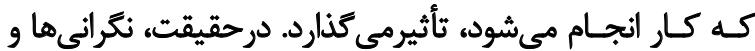


را به خطر اندازد و موجب نارضايتى اربابرجوع شود [IV]]

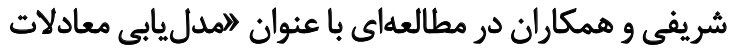

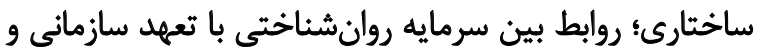

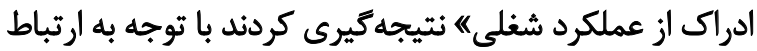

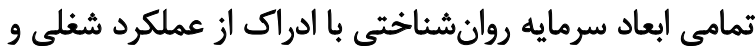

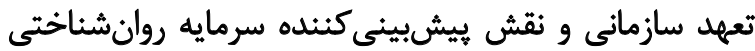

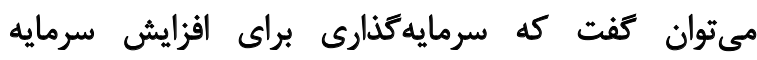

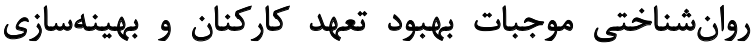

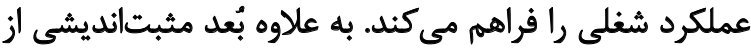

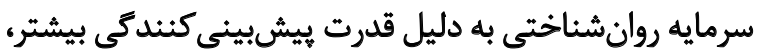
قابليت تأمل بيشترى دارد [1/]]. اندام و همكاران در يثوهشى درباره ارتباط بين اخلاق كارى،

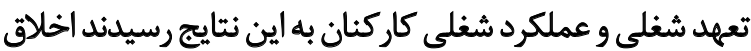

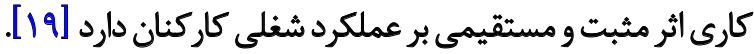

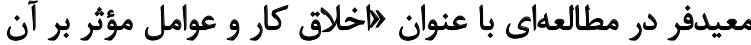

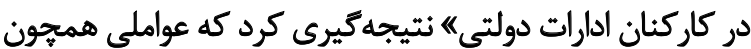

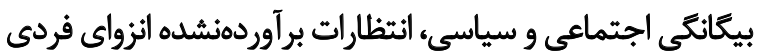

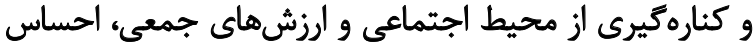

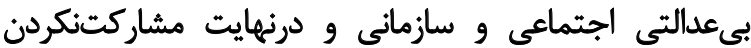

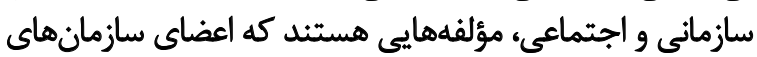

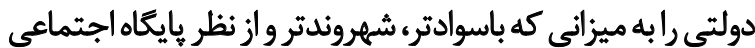

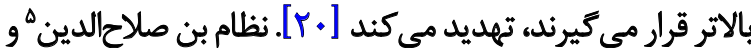

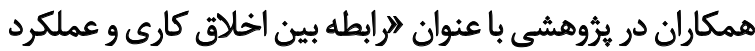

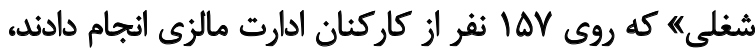

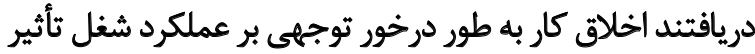

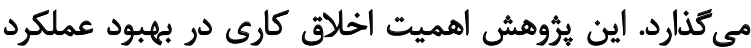

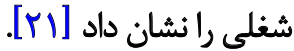

از آنجا كه دانشكاه علوم بهزيستى و توانبخشى و مراكز تابعه

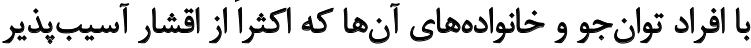

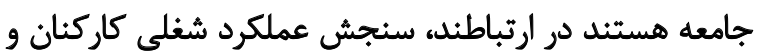

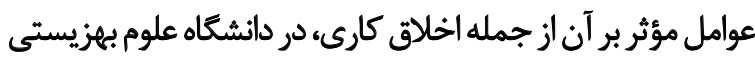

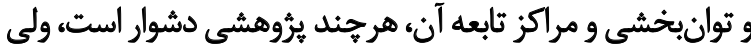

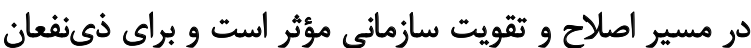

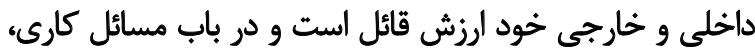
كاركنان را تشويق مي كند. كاركنان ستادى و و عملياتي دانشكاه

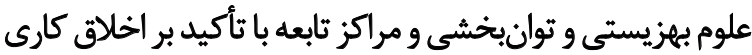

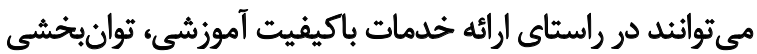

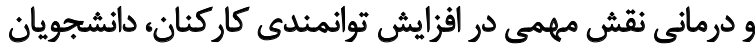

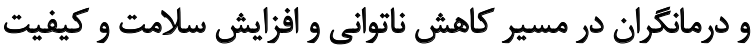

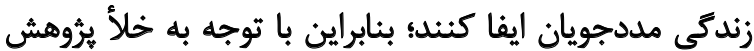
در زمينه اخلاق كار و رابطه آن با عملكرد شغلى در دائ دانشعاه
به اخلاق كارى توجه ميشود. در حالى كه در غرب سكولارّ".

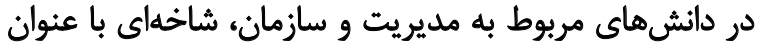

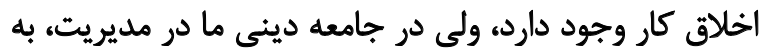

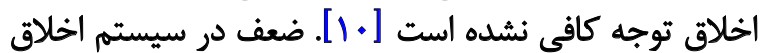

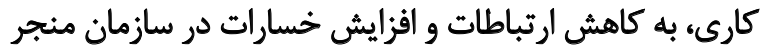

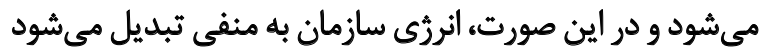

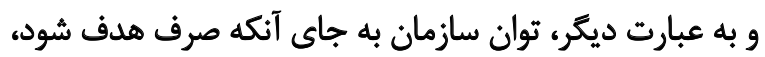

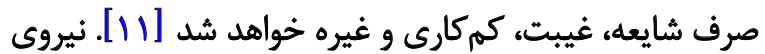

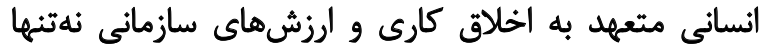

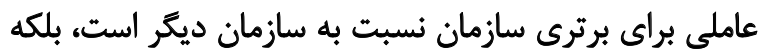

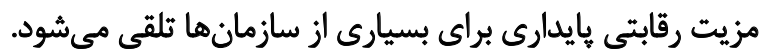

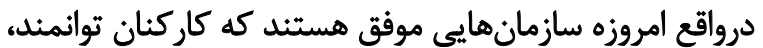

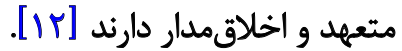

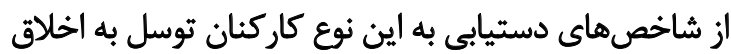

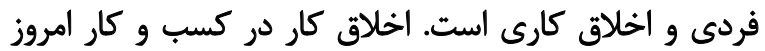

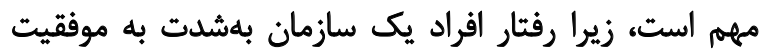

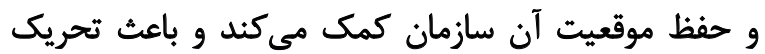

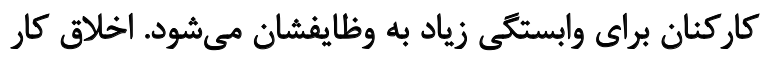

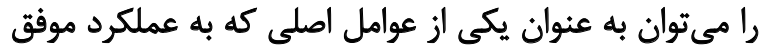

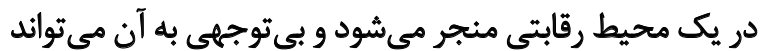

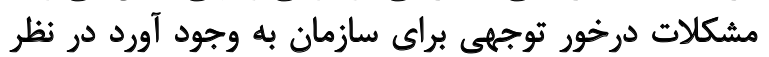

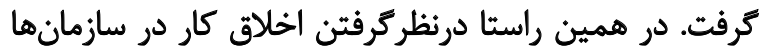

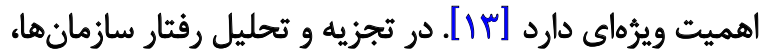

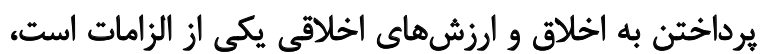

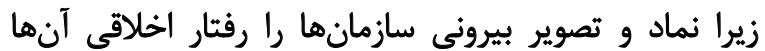

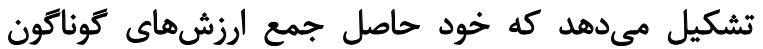

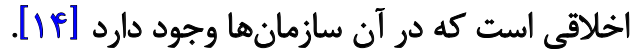

بيىتوجهى سازمانها به اخلاق كار و ضعف در رعايت اصول

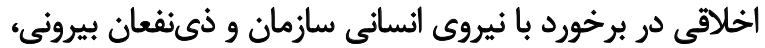

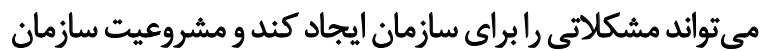

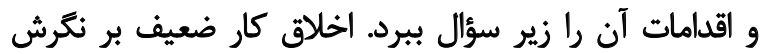

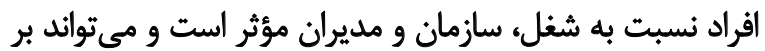

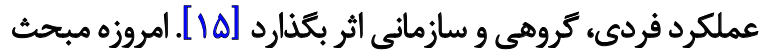

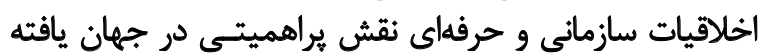

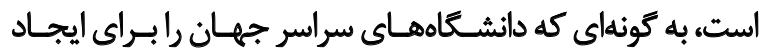

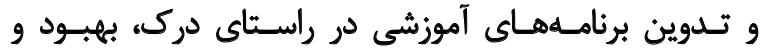

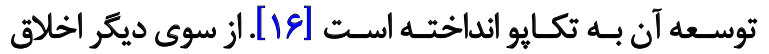

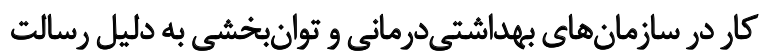

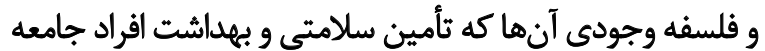
است، اهميت بيشترى، نسبت به ساير ساير سازمانها دانها دارد. انجام

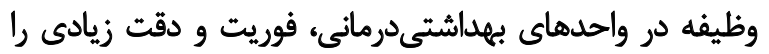

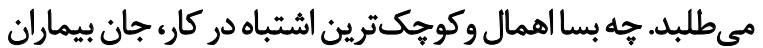




$$
\text { روح جمعى و مشاركت دركار (^ يرسش). }
$$

در تحقيق انجامشده از سوى يتى در سال سوادادر

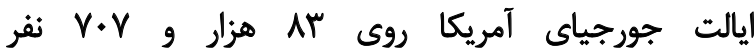

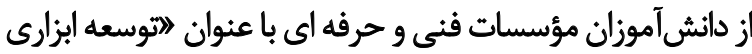

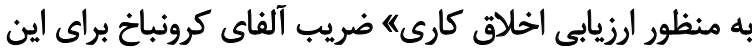

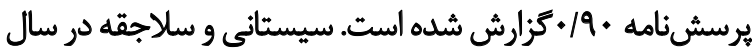

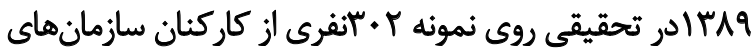
دولتى شهر كرمان با عنوان البررسى رابطه ميان اخلاق كارئ بان و

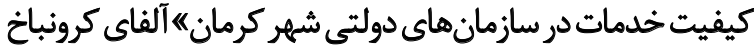

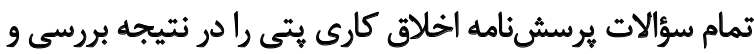

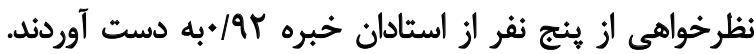

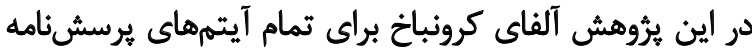

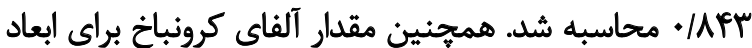

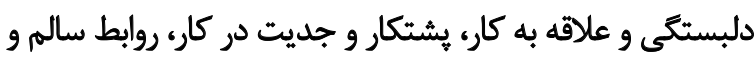

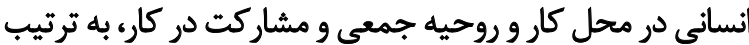

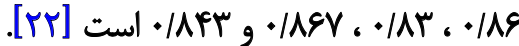

به منظور سنجش عملكرد شغلى كاركنان از يرسشناميه

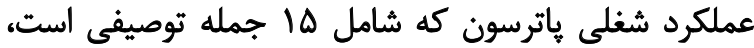

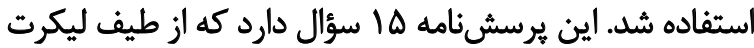
جهارتايى از صفرتا ب تشكيل يافته است.

اين يرسشنامه زيرمقياس ندارد و براى محاسبه عملكرد

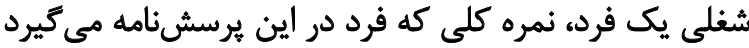

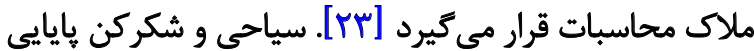

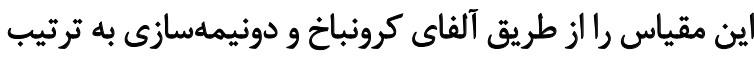

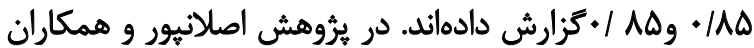

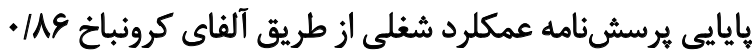

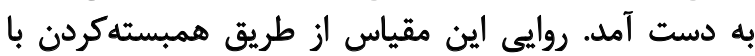

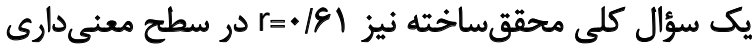

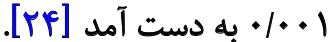

بعد از ارائه توضيح و كسب رضايت از شركت كنيندكان در

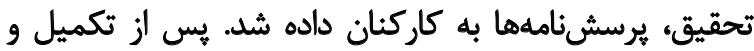

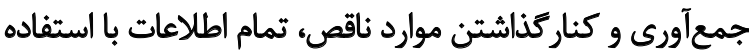

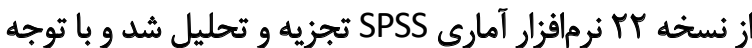

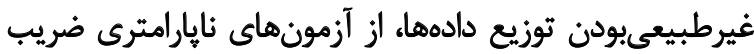

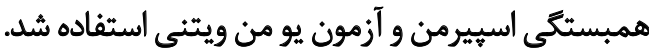

يافتثهها

بررسى مشخصات جمعيتشناختى تحقيق نشان داد ميانكين

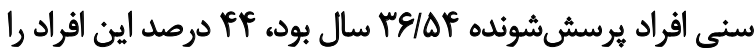

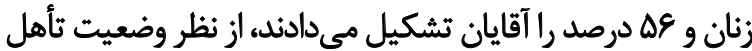

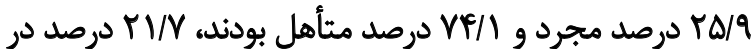

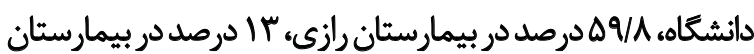

علوم بهزيستى و توانبخشى و مراكز تابعه اين مطالعه با هدف

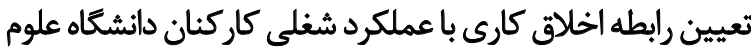

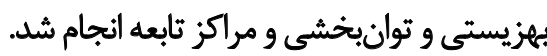

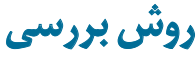

اين يُروهش از لحاظ هدف در دسته يروهش هاى كاربردى وازي واز

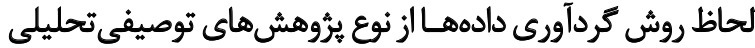

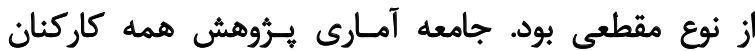

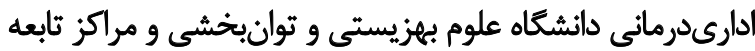

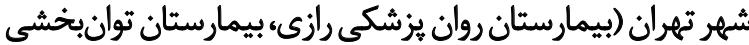

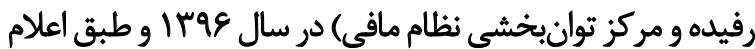

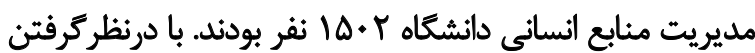

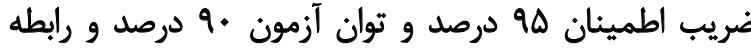

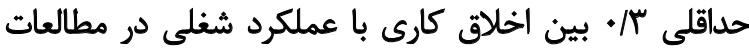

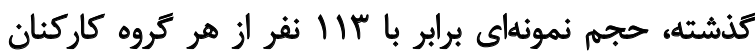

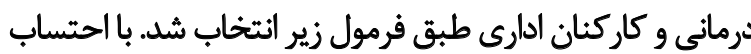

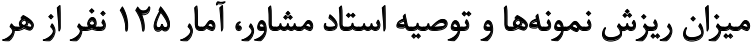

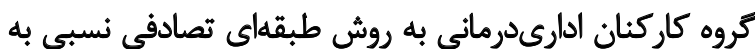

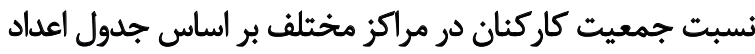

$$
n=\frac{\left(z_{1-\frac{a}{2}} z_{1-6}\right)^{2}}{\left(\frac{a}{2} \ln \left(\frac{1+r}{1-r}\right)^{2}\right.}+3=113
$$

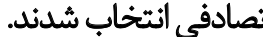

$$
\begin{aligned}
& z_{1-\frac{a}{2}}=1.96 \\
& r=0.35 \\
& z_{1-6}=0.84
\end{aligned}
$$

معيار ورود نمونهها عبارت بود از: همه كاركناني كه مدرك

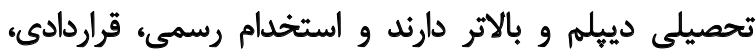

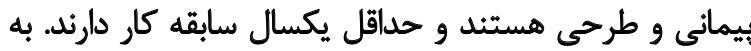

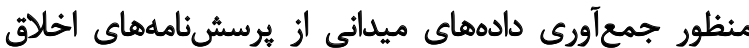

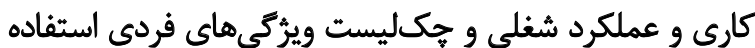

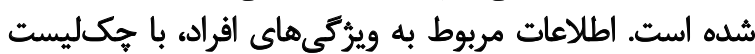

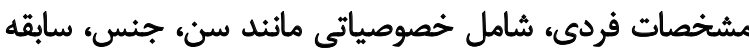

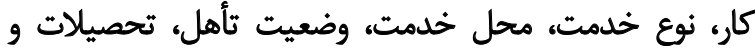
وضعيت استخدامى، جمع آورى شد.

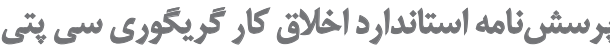

مورسنامه اخلاق كار را دكتر كريكورى سى يتى در سال

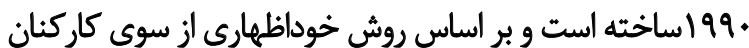

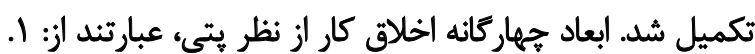

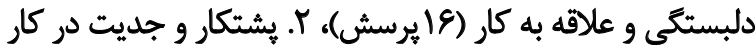

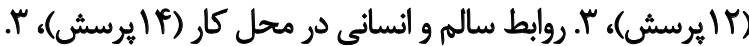


جدول ا. رتبههاى عملكرد شغلى كاركنان به تفكيك اطلاعات جمعيتشناختى

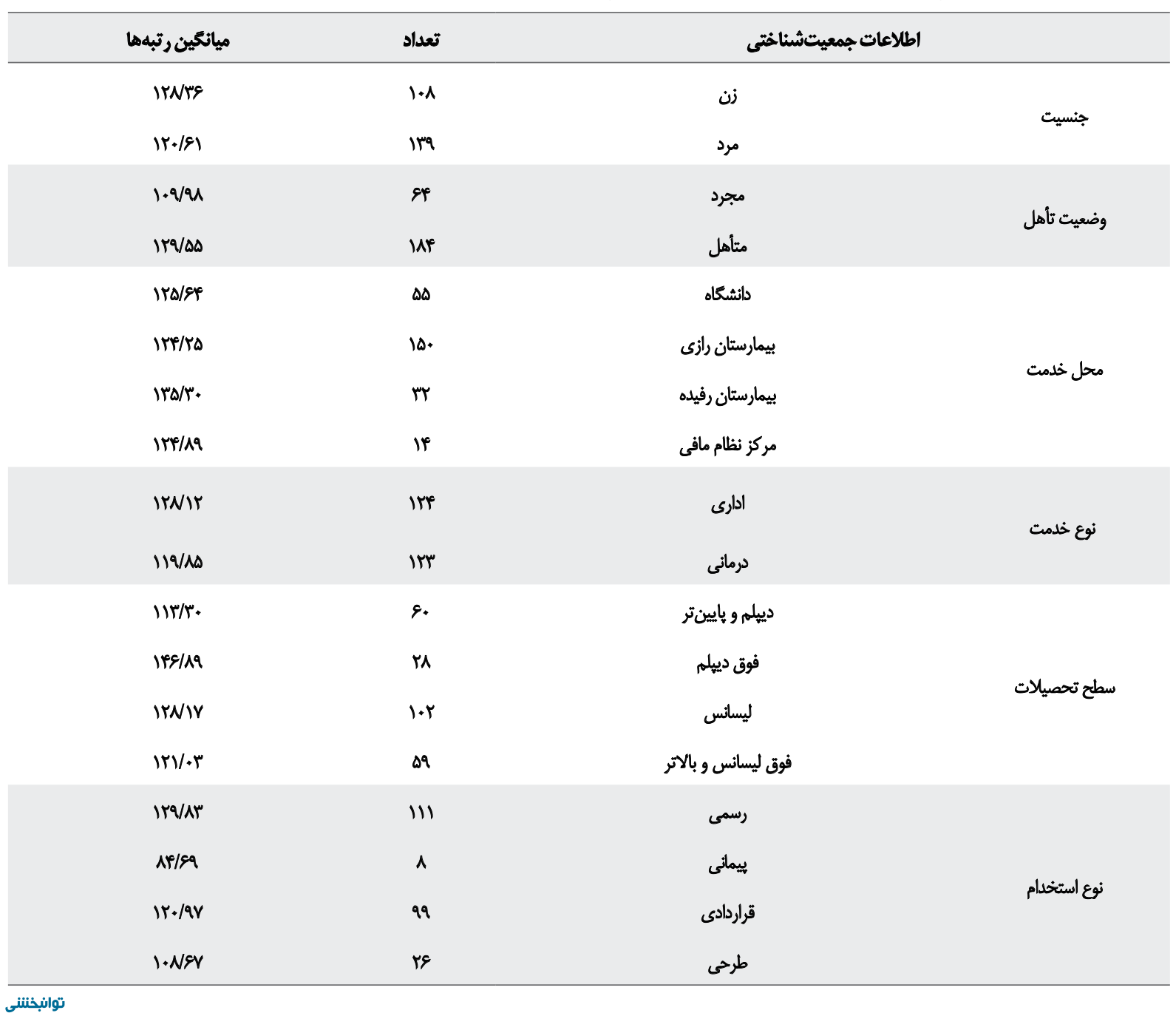

رفيده و ه/ه درصد در مركز نظام مافي خدمت مي كردند.

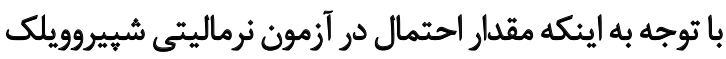

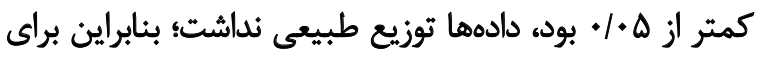

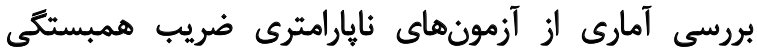

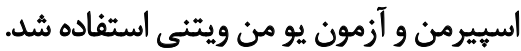

همانطور كه در جدول شماره r ملاحظه مي شود، مقدار

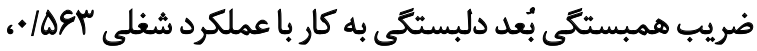

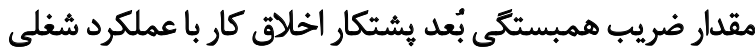

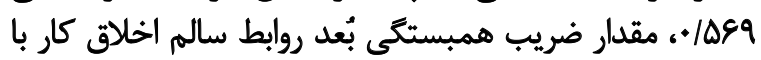

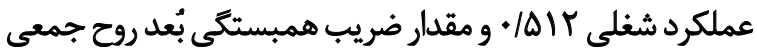

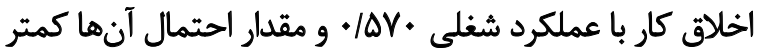

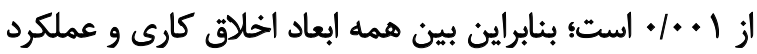

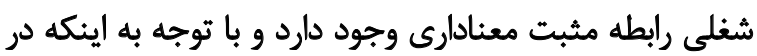

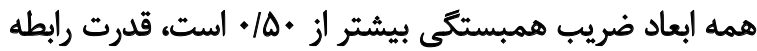

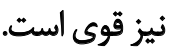

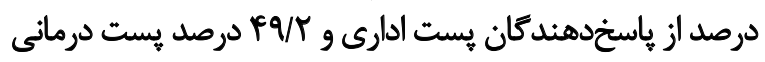

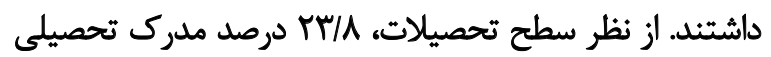

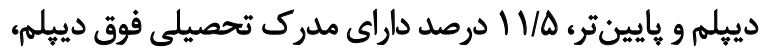

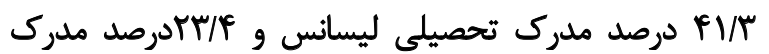

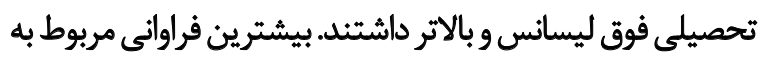

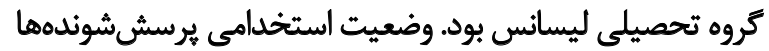

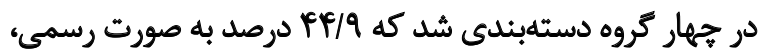

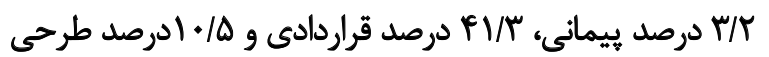

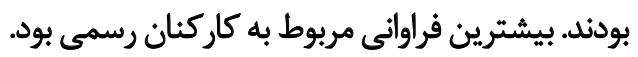
همانطور كه در جدول شماره ا مشاهده مىشود، ميانگين

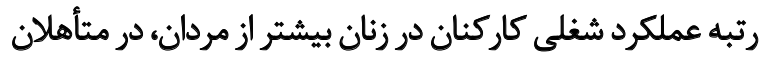

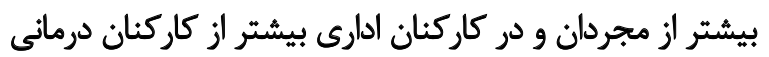

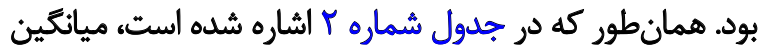

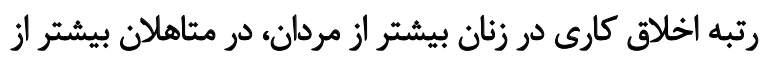

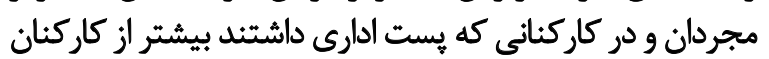


جدول ץ. رتبهاى اخلاق كارى به تفكيك اطالاعات جمعيثشئنتى

\begin{tabular}{|c|c|c|c|}
\hline ميانكين رتبهها & تعداد & اطلاعات جمعيتششناختى & \\
\hline ITV/RT & $1 \cdot 1$ & is & \multirow{3}{*}{ جنسيت } \\
\hline & & & \\
\hline IT/T/ & 119 & مرد & \\
\hline$\| A / T E$ & er & هجرد & \multirow{2}{*}{ وضعيت تأهل } \\
\hline & & هتtأه| & \\
\hline IYNEE & $\Delta \Delta$ & كانشكاه & \multirow{5}{*}{ همل خدمت } \\
\hline $\mid r \Delta / * A$ & 10 & بيمارستان رازى & \\
\hline & & & \\
\hline 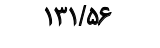 & $\pi$ & بيمارستان رفيله & \\
\hline $\mid r \Delta / / H$ & if & هركز نظام مافى & \\
\hline$I M / \Delta$. & inf & الدارى & \multirow{3}{*}{ نوع خلمت } \\
\hline & & & \\
\hline $118 / 18$ & IMT & درمائى & \\
\hline Ire/ve & 8. & دييلم و يايينتر & \multirow{5}{*}{ سطح تحصيلات } \\
\hline IMT/gY & ra & فوق دييلم & \\
\hline & & & \\
\hline ITVIAY & $1 . r$ & ليسائس & \\
\hline Ire/a & $\Delta q$ & فوق ليسانس و بالاتر & \\
\hline ITV/TQ & 111 & رسمى & \multirow{5}{*}{ نوع استخدام } \\
\hline $1.1 / 8 \pi$ & $\wedge$ & يبيمائى & \\
\hline & & & \\
\hline$M T / T \Delta$ & 99 & قراردادى & \\
\hline WWFF & re & طرحى & \\
\hline
\end{tabular}

توانبخننى

شغلى افراد متأهل بيش از افراد مجرد است كه با يافتهاي

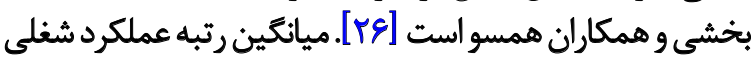
كاركنان بيمارستان رفيده از ساير مراكز بيشتر استر استه ميانكين

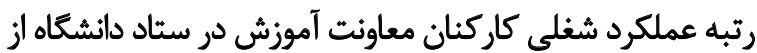

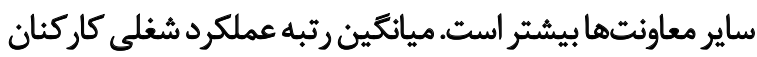

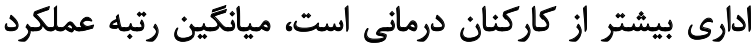

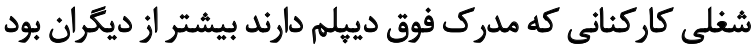

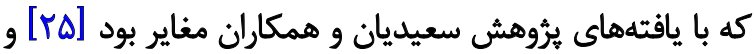

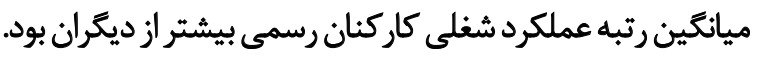

اين يُروهش با هدف تبيين رابطه اخلاق كارى با عملكرد شغلي

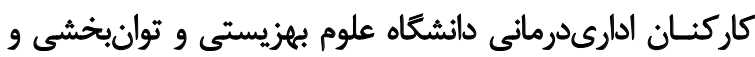

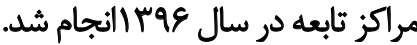

نتايج اين يروهش درباره رابطه بين اطلاعات جمعيتشناختى

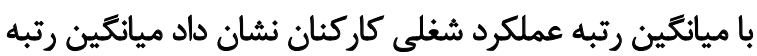

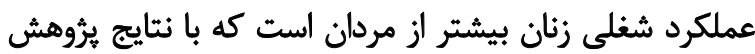

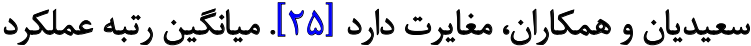

\begin{tabular}{|c|c|c|c|c|}
\hline روح جمعى و مشاركت در كار & روابط سالم در كار & يشتكار و جديت در كار & دلبستكى و علاقه به كار & عملكرد شغلى \\
\hline.$/ \Delta V$ &.$/ \Delta 14$ & .1859 &. & ضريب همبستكى \\
\hline $.1 . .1$ &.+1 & $.1 .+1$ & $.1+1$ & مقدار احتمال \\
\hline
\end{tabular}

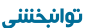


تلطيف مىشود و بر كيفيت عملكرد آنها مىافزايد. تحليل يافتههاى يُروهش نشان داد اخلاق كارى بر عملكرد

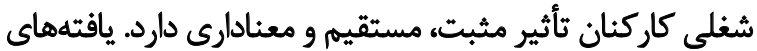

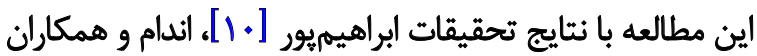

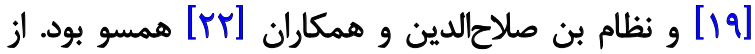

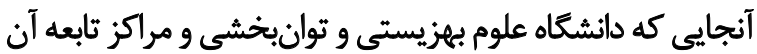

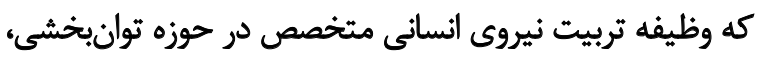

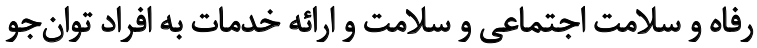

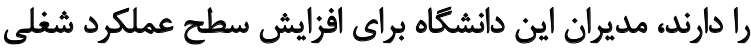

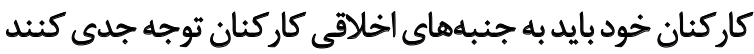

و سعى در افزايش سطح اخلاقى كاركنان خود داشته بارئن باشند.

نتيجهيرى

تجزيه و تحليل نهايى يافتههاى يروهش نشان داد اخلاق كارى

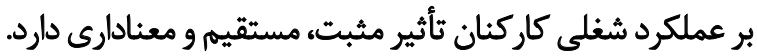

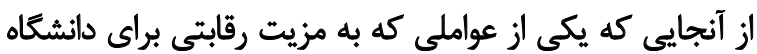

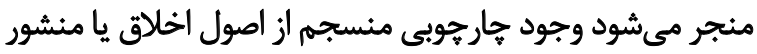

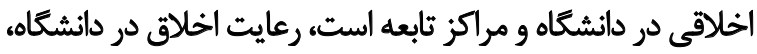

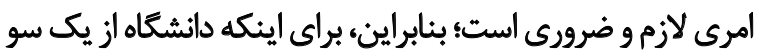

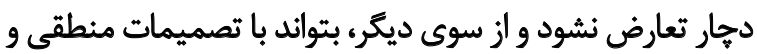

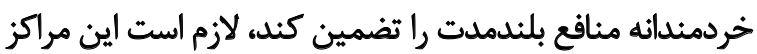

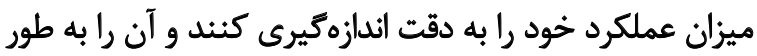

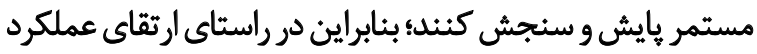

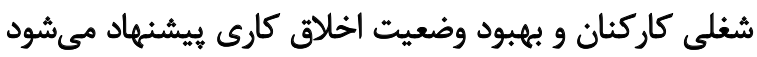

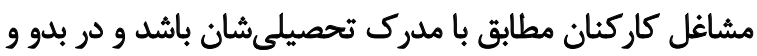

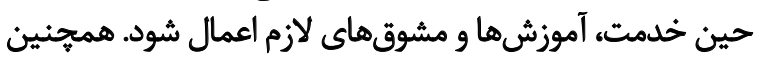

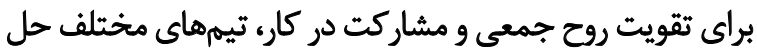

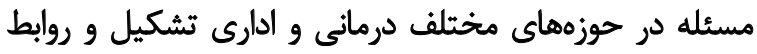

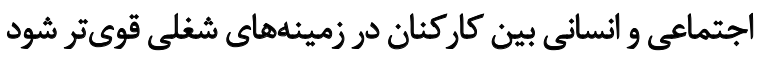

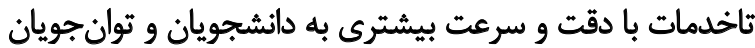
ارائه شود.

از محدوديتهاى اين مطالعه مي توان به نبود همكارى مناسب

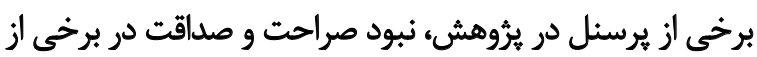

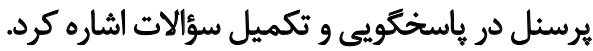

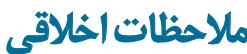

\section{يبيروى از اصول اخلاق يُؤهش}

كميته اخلاق دانشكاه علوم بهزيستى و توانبخشى اين مطالعه

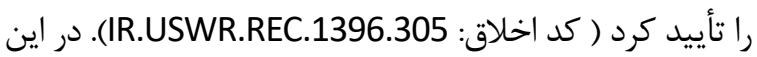
يروهش به تمام شركتكنند براى شركت در مطالعه از آنها موافقت آكاهانه كَرفته شد.
همجينين نتايج اين يرؤهش درباره رابطه بين اطلاعات

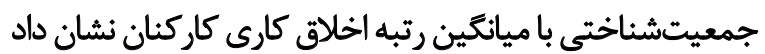

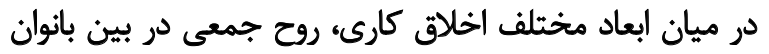

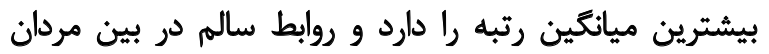

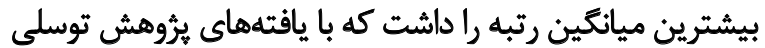

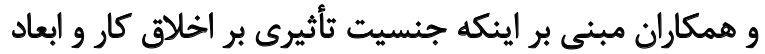

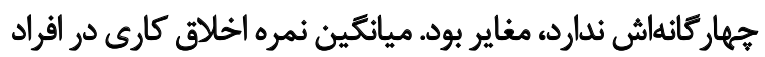

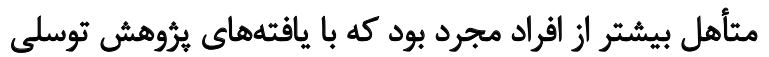

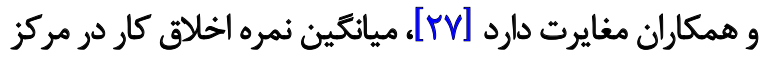

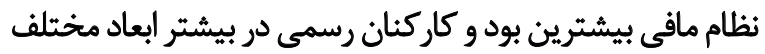

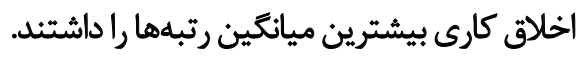

بر اساس نتايج بهدست آمده از اين بُروهش، بين بُعد دلبستَّى

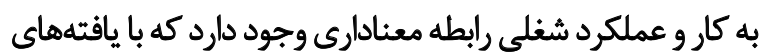

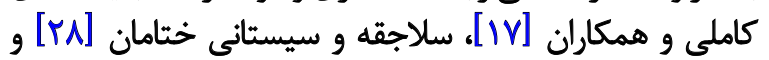

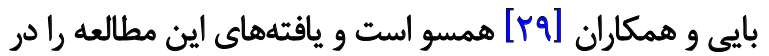

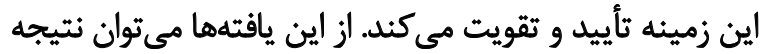

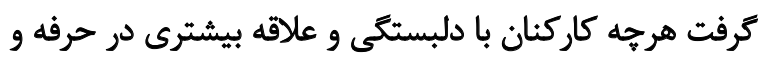

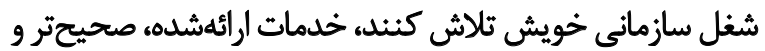

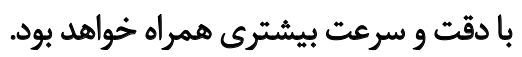

در اين يرؤهش بين بُعد يشتكار و عملكرد شغلى رابطه

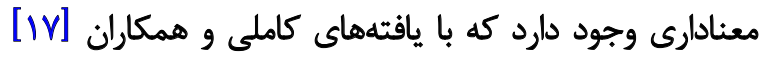

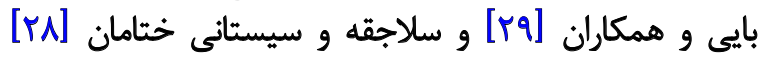

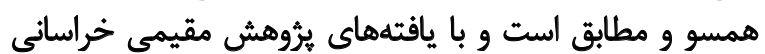

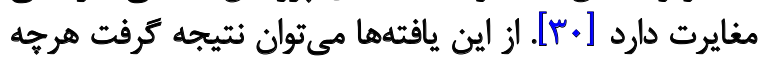

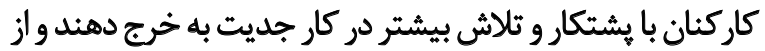

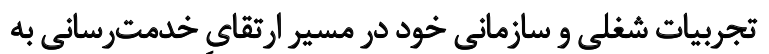

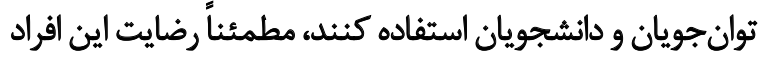

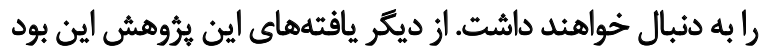

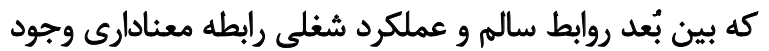

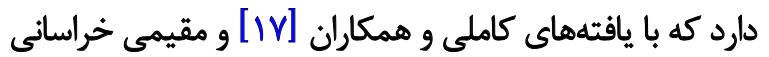

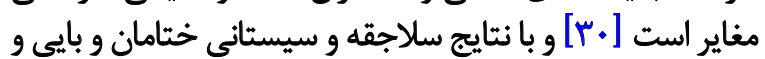

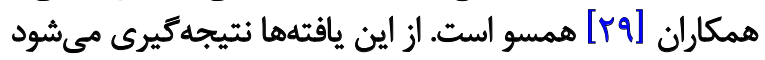

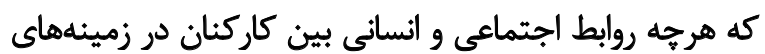

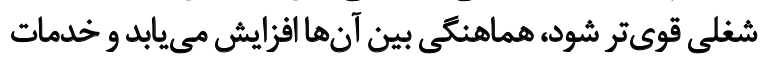

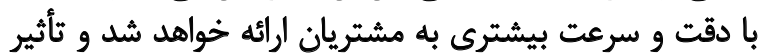

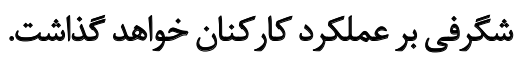

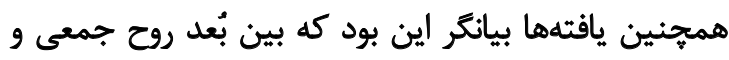

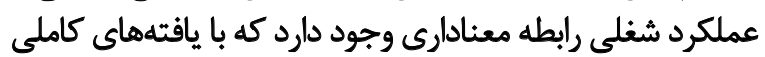

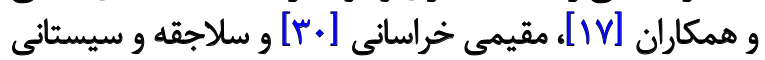

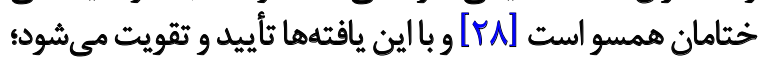

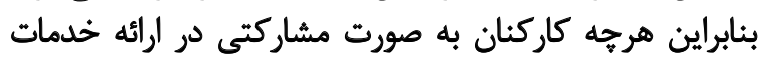

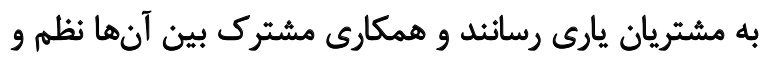

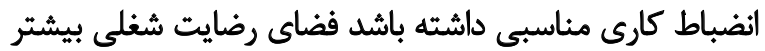




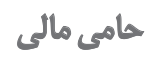

اين مقاله بخشى از باياننامه نويسنده اول در مقطع كارشناسى

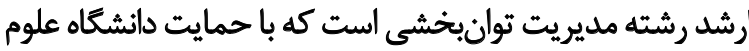
بهزيستى و توانبخشى اجرا شده است.

$$
\text { مشار كث ثويسندكان }
$$

مشاركت نويسندكان به شعح زير است: ايدهازى:

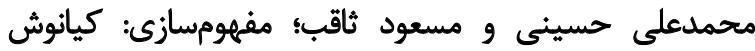

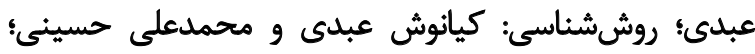

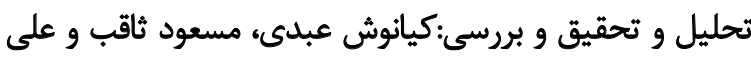

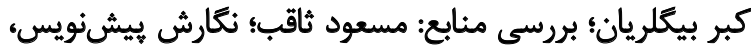

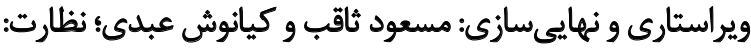

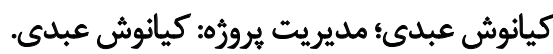

$$
\text { تمارض منافع }
$$

بنابر اظهار نويسندكان، اين مقاله تعارض منافع ندارد.

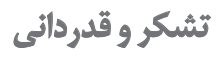

نويسندكان بر خود لازم مى دانيد از رياست دانشكاه و مديران ستادى و مديران مراكز تابعه دانشكاه و كاركنان ادارئ دارى درمانى دانشكاه علوم بهزيستى تشكر كنيند. 


\section{References}

[1] Ghahramani M, Abolghasemi M, Arefi M, Khatami M. [Examine organizational health at Shahid Beheshti University (Persian)]. Quarterly Journal of Career \& Organizational Counseling. 2012; 4(10):13-30.

[2] Barati Ahmadabadi H, Oreizi HR, Nouri A. [Relationship between organization climate and work conscientiousness with job performance (Persian)]. Journal of Applied Psychology. 2010; 4(1):81-65.

[3] Ziyae B, Mohammad Mobaraki MH, Saeediyoun M. [The effects of psychological capital on organizational performance with an emphasis on the mediatory role of innovation in information technology: A case study of the branches of agriculture bank in Tehran (Persian)]. Innovation Management. 2014; 3(4):77-100

[4] Jalali A, Rohollahi A. [The impact of transformational leadership on the job function of the faculty with the mediation of job conf (Persian)]. Journal of Public Administration. 2016; 6(17-18):1-16.

[5] Kavousy K. [Assessing the relationship between social capital and occupational activity (Case of study: employees in Islamic Azad University East Tehran Branch) (Persian)]. Iranian Social Development Studies. 2015; 7(4):85-104.

[6] Ahadi B, Fathi A, Abdolmahmadi K. [Investigating psychological factors affecting job performance of police command staff (Persian)]. Journal of Law Enforcement, East Azarbaijan. 2014; 4(15):78-63.

[7] Sajjadi N, Omidi A. [Factors influencing job performance of staff experts of P.E. Organization (Persian)]. Harakat. 2009; 38:81-93.

[8] Taheri Attar G, Naieni MA, SeydSalehi SS, Khezri A. [Ethical leadership facilitates the realization of good governance (Case study: Tehran municipality) (Persian)]. Journal of Public Organizations Management. 2017; 6(1):115-30.

[9] Khayat Moghadam S, Tabatabaei Nasab M. [Components of professional ethics in management (Persian)]. International Journal of Ethics and Society. 2016; 11(1):127-36.

[10] Taheri Z. [Relationship between work ethic and staffs, quality of services gap rehabilitation centers in Isfahan welfare organization (Persian)] [MSc. thesis]. Tehran: University of Social Welfare and Rehabilitation Sciences; 2015.

[11] Bayat M. [Explaining professional ethics in the organization (Persian)]. Paper presented at the Third International Conference on Accounting and Management; 2 November 2014; Tehran, Iran.

[12] Enayati T, Behnamfar R, Esfandiari S. [The relationship between work ethics and organizational commitment among nurses in hazrat Fatemeh Zahra Hospital in Sari (Persian)]. Breeze of Health. 2014; 2(2):50-5.

[13] BakhshChenari A, Eydi H, Abbasi H. [Analyze relationship between organizational forgetting and agility with organizational performance (Case study: Yoths and Sport Ministry) (Persian)]. Organizational Behavior Management in Sport Studies. 2015; 13(4):96-87.
[14] Khoddami S, Osanloo B. [Designing ethical behavior model of employees with emphasis on the role of ethical values of organization (Persian)]. Ethics in Science and Technology. 2015; 10(1).

[15] Niazazari K, Enayati T, Behnamfar R, Kahroodi Z. [Relationship between professional ethics and job commitment (Persian)]. Iran Journal of Nursing. 2014; 27(87):34-42. [DOI:10.29252/ijn.27.87.34]

[16] Sheikhzakaryaie N, AtashzadehShoorideh F. The relationship between professional ethics and organizational commitment of faculty members in Kurdistan University Of Medical Sciences. Advances in Nursing \& Midwifery. 2016; 25(91):21-30.

[17] Kameli M, Zabet M, Mohebbi K. [A survey on the relationship between job ethics and job performance among employees in the Abhar healthcare network (Persian)]. Development of Human Resources Management and Support. 2015; 36(10):17-34

[18] Sharifi NS, Shahtalebi B. [Investigation of the relationship between dimension of psychological capital with organizational commitment and job performance (Persian)]. Social Welfare. 2015; 15(59):119-48.

[19] Andam R, Montazeri A, Abavisani A. [The relationship between work ethic, job commitment and job performance among employees of the offices of sport and youth in Khorasan Razavi Province (Persian)]. Contemporary Research in Sport Management. 2015; 5(10):61-72

[20] Moeidfar S. [Work ethics among state office employees of the Tehran Provincial administration (Persian)]. Social Welfare. 2007; 6(23):321-44.

[21] Salahudina SNb, Alwia MNRb, binti SS, Baharuddina, Halimata SSb. The relationship between work ethics and job performance. Paper presented at: $3^{\text {rd }}$ International Conference on Business and Economics. 21-23 September 2016; Johor Bahru.

[22] Sezavar H, Kavari H, Hatamizadeh N, Torabi S. [Investigating the status of work ethics among rehabilitation-therapeutic centers of Tehran University of Social Welfare and Rehabilita tion Sciences in 2013 (Persian)]. Woman and Family Studies. 2011; 6(21):87-101.

[23] Vosoughi Niri A, Ruhollahi A, Mohmmad Hosein H. [A survey of effect of job stress on general health and job performance on Air Traffic Controllers (ATC) (Persian)]. Iran Occupationa Health Journal. 2016; 13(1):47-57.

[24] Aslanpoor JM, Shahbazi F, Maleki R. [The relationship between vigor, health and job performance in employees Of The Industrial Company, Ahvaz (Persian)]. Journal of Social Psychology. 2012; 7(21):65-73.

[25] Saeedian H, Mahmoodi M, Haghighat S. Comparison of job performance of male and female employees of Fars Telecommunication Company. Paper presented at: $2^{\text {nd }}$ International Conference on Humanities, Psychology and Social Sciences. 15 March 2016; Istanbul, Turkey.

[26] Bakhshi E, Kalantari R. [Investigation of quality of work life and its relationship with job performance in health care workers (Persian)]. Journal of Occupational Hygiene Engineering 2017; 3(4):31-7. [DOI:10.21859/johe-03044] 
[27] Tavassoli G, Nahavandi M. [A study on work ethic (Persian)]. Women in Development \& Politics. 2008; 6(3):39-60.

[28] Sistani F, Salajighe S. [Investigating the relationship between work ethics and service quality in government organizations in Kerman (Persian)]. Paper Presented at: National Conference on Management and Leadership Challenges in Iranian Organizations. 24 July 2010; Isfahan, Iran.

[29] Ahadollah B, Jvad M. [Relationship between job ethics and job performance of employees at the institute of fisheries re-

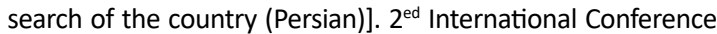
on Accounting, Economics and Financial Management. 18 June 2016; Shahrekord, Iran.

[30] MoghimiKhorasani A. [Relationship between work ethic and creativity of employees (Persian)]. Ethics in Science and Technology. 2016; 10(3):92-85. 
This Page Intentionally Left Blank 